\title{
Real-time model of switched reluctance drive based on a LUT magnetization curve for educational purposes
}

Savkin, D., Anuchin, A., Kulmanov, V., Vagapov, Y. and Gulyaev, I.

This is a paper presented at the 2017 15th International Conference on Electrical Machines, Drives and Power Systems (ELMA)

Copyright of the author(s). Reproduced here with their permission and the permission of the conference organisers.

\section{Recommended citation:}

Savkin, D., Anuchin, A., Kulmanov, V., Vagapov, Y. and Gulyaev, I. (2017), 'Real-time model of switched reluctance drive based on a LUT magnetization curve for educational purposes', in Proc. 15th IEEE Int. Conf. on Electrical Machines, Drives and Power Systems ELMA2017, Sofia, Bulgaria, 1- 3 June 2017, pp. 480-483. doi: 10.1109/ELMA.2017.7955491. 


\title{
Real-time Model of Switched Reluctance Drive Based on a LUT Magnetization Curve for Educational Purposes
}

\author{
Dmitrii Savkin ${ }^{1}$, Alecksey Anuchin¹, Vasiliy Kulmanov ${ }^{1}$, Yuriy Vagapov², Igor Gulyaev \\ ${ }^{1}$ Electric Drives Department Moscow Power Engineering Institute, Moscow, Russia, savkindmi@gmail.com \\ ${ }^{2}$ School of Applied Science, Computing and Engineering Glyndwr University, Wrexham, UK, y.vagapov@glyndwr.ac.uk \\ ${ }^{3}$ Electronics and nanoelectronics Department, Ogarev Mordova State University, Saransk, Russia, ivgulyaev@mail.ru
}

\begin{abstract}
This paper discusses the implementation of a realtime model of a switched reluctance motor drive for which the parameters of its magnetisation curve are represented by the data in a look-up table. A method of cubic interpolation has been applied for the look-up table approximation in order to provide a high accuracy and realistic performance of the modelled motor drive. The model structure has been optimised for real-time execution in a motion control microcontroller operating under PWM frequency up to $40 \mathrm{kHz}$. The model is designed to be used in the students' coursework on the specialist subject of electric drive control.
\end{abstract}

Keywords-Variable speed drives; Electrical engineering education; Power system modeling; Microcontrollers; Switched reluctance drive

\section{INTRODUCTION}

Engineering coursework is usually a problem solving exercise in which students are involved in self-directed problembased learning and it is a fundamental component of an engineering curriculum, based on student-centred teaching strategies across the program. The major learning outcome of coursework is to develop the students' ability to apply their practical skills for real-world problem solving [1], [2].

The coursework on the specialist subject of Electric Drive Control discussed in this paper reflects recent advances in the area of digital technology which dominates in modern control engineering. The content of the coursework focuses on the development of a control system for various types of electric drives using modern microcontrollers designed for industrial and domestic motion control applications.

A typical coursework problem requires a student to investigate, develop, implement and test a control algorithm for a particular type of electric motor drive which has been selected for each student individually. The completed algorithm is a software code comprising a set of instructions for the microcontroller to provide appropriate control of the selected motor drive. For safety reasons, the electric drive to be controlled by the algorithm is a virtual model integrated into the same microcontroller and operating in a real-time mode simultaneously with the student's control algorithm code [3].

The motor drive model is provided to the students as a binary library for $\mathrm{C}$ language. It is proposed that the Code Composer
Studio integrated development environment will be used for the control system design and debugging.

However, according to the coursework task there is a problem solving component in the learning activity i.e. the motor drive parameters represented by the binary library are unknown for the student and must be determined at the initial stage of the software development. Therefore, it is expected that the student will conduct a series of virtual experiments to investigate the motor drive parameters in order to build a desired control system.

The library of the drive models for the coursework currently includes three types of motors: DC motors, induction machines and a family representing permanent magnet synchronous machines, synchronous reluctance machines and switched reluctance machines (SRM). It has been observed that the area of applications for SRM drives has rapidly expanded over the past decades [4], [5] which makes SRM extremely attractive for the electric drive design, research and study. As for the previously discussed coursework, the model of SRM is represented using a linearised magnetisation curve with a sharp saturation knee [6] (Fig. 1a). Such a rough approximation has been done in order to simplify the numerical calculations of the model, which should execute and operate in a real-time mode. However, the results obtained from this model execution are quite different in some areas compared to the performance of a real SRM as shown in Fig. 1b. The red circles in Fig. 1b mark the instant change of the current derivative due to a sharp change of the inductance in the phase at the saturation point.

Computational resources of modern microcontrollers can provide much faster software execution and increase the complexity of the equations for numerical integration. Due to the advanced specification of state-of-art microcontrollers the SRM model structure has been significantly revised in order to improve the accuracy of the magnetisation curve representation. It has been suggested that the magnetisation curve could be defined by a look-up table [LUT] containing the data linked to the SRM flux linkage, phase current and rotor position. In order to reduce the computational load the data is processed at the model initialisation stage in order to be appropriately integrated into the numerical equations of the motor model. 


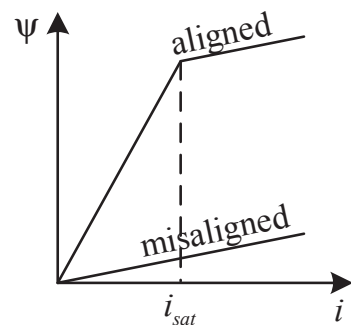

a)

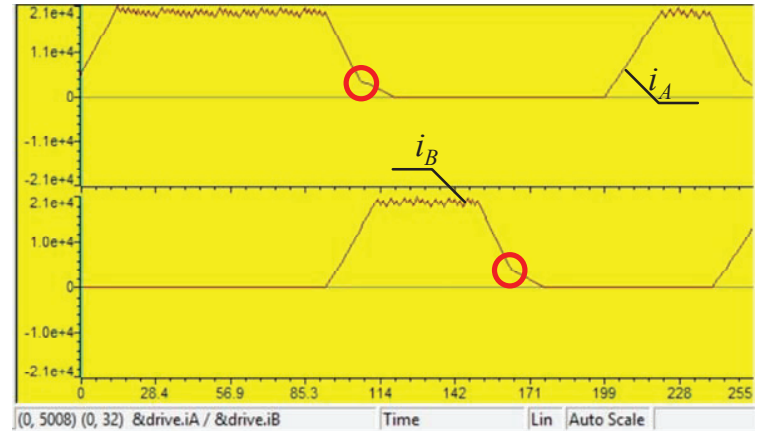

b)

Fig. 1. Simplified magnetisation curve (a) and operation of the SRM model (b).

\section{IMPLEMENTATION OF SWITCHED-RELUCTANCE DRIVE MODEL}

\section{A. Model User Interface and Inverter Topology}

The model of the switched reluctance drive contains a motor and an inverter. The topology of the inverter and the connection diagram are shown in Fig. 2.

The model is driven by variables, which can be set by a student in his control algorithm. The PWM period is defined in variable drive.tpr and represented in CPU clock cycles (1/150,000,000 s for $150 \mathrm{MHz}$ microcontroller). The duty cycle for each phase bridge are defined in variables drive.cmpr1, drive.cmpr 2 and drive.cmpr 3 for each phase of the motor. These duty cycles are applied for both switches of each phase bridge. Also the student can apply load torque to the motor shaft by changing variable drive.load. The model is designed to provide the current and position feedbacks for further processing. The current feedback readings (drive.iA, drive.iB, drive.iC) are given in ADC scale. The position feedback is represented by the readings of the tachogenerator (drive.adcSpeed) measuring the speed directly, the Hall sensor (drive.hallSensor contains a binary position code) and the incremental encoder (drive.qepCounter).

\section{B. Representation of the Magnetisation Curve}

An SRM is a non-linear motor where the effect of saturation is used to increase the motor efficiency. The previous SRM model was based on the magnetisation curve linearised using two zones located below and above the saturation current point as shown in Fig. 1a. Although, under this approach, the sharp knee of the curve introduces the observed instant change of the current derivative, the model equations are very simple and can be calculated by microcontroller in real-time mode.

The motor manufactures can provide magnetisation surface or magnetisation curves for both aligned and misaligned positions. The magnetisation curve is mandatory if the motor has an asymmetrical rotor configuration, for instance, two phase machines are designed so as to reduce the number of switches in the inverter and to ensure that the motor can start rotating in the right direction. For three- and more phase machines the rotor is usually symmetrical and only two curves for aligned and misaligned positions are enough for relatively accurate representation as shown in Fig. 3. Supposing that the flux linkage is varying in accordance with a sinewave from the aligned to misaligned position the remaining part of the flux linkage surface can be interpolated by the following equation:

$$
\begin{aligned}
& \psi=\frac{\psi_{\text {aligned }}(i)+\psi_{\text {misaligned }}(i)}{2}+ \\
& +\frac{\psi_{\text {aligned }}(i)-\psi_{\text {misaligned }}(i)}{2} \cos \theta
\end{aligned}
$$

where $\psi_{\text {aligned }}(i)$ and $\psi_{\text {misaligned }}(i)$ are the functions of flux linkages for aligned and misaligned positions, $\theta$ is the electrical rotor position. The curves of flux linkages can be represented as look-up tables with variable step between points.

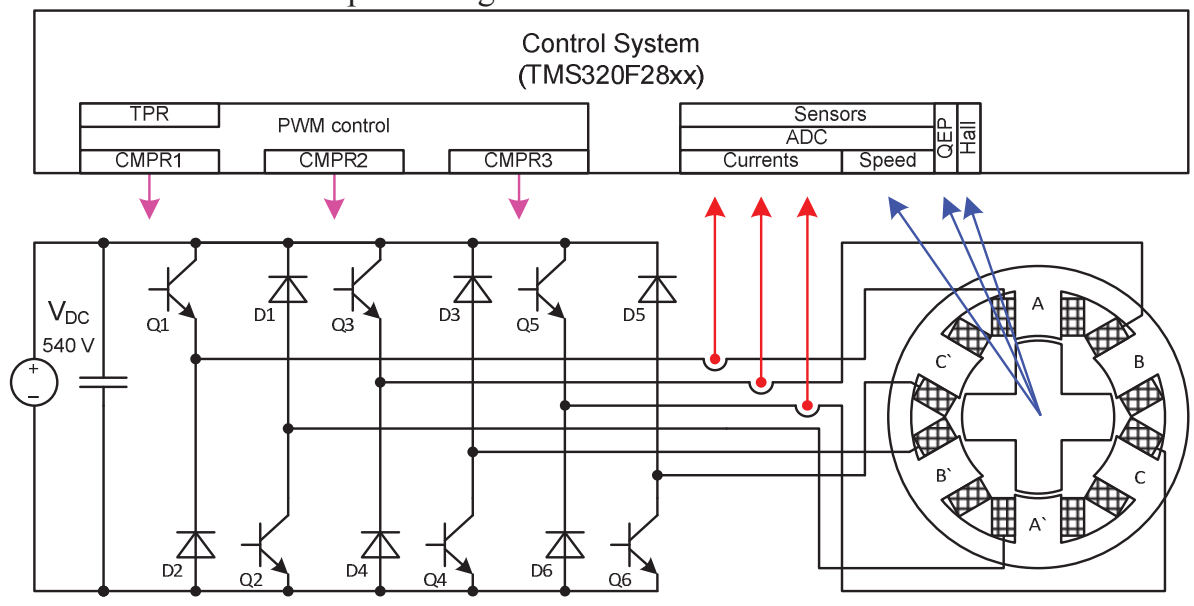

Fig. 2. Functional diagram of the switched reluctance drive model. 


\section{Motor equations}

Taking into account that each phase of the motor is driven individually and the majority of motors are designed with minimal mutual inductance between the phases, then the mutual inductance can be disregarded. The equation of the phase is:

$$
v=i R+\frac{d \psi}{d t}
$$

where $v$ is the applied phase voltage, $i$ is the phase current, $R$ is the stator resistance and $\psi$ is the flux linkage.

The flux linkage and the current are connected with the magnetisation surface. The torque of the machine can be defined as a function of the phase energy:

$$
T=-p_{p} \frac{\partial \int_{0}^{\psi} i(\theta, \psi) d \psi}{\partial \theta}
$$

or co-energy:

$$
T=p_{p} \frac{\partial \int_{0}^{i} \psi(\theta, i) d i}{\partial \theta}
$$

where $p_{p}$ is the number of pole pairs of the motor [7].

\section{Model Calculation Algorithm}

The first step of the model algorithm is the voltage balance equation written in the Cauchy form for numeric integration:

$$
\Delta \psi=(v-i R) T_{P W M}
$$

where $T_{P W M}$ is the duration of PWM cycle or the integration step size.

The known flux linkage can be used to evaluate the phase current using the magnetisation surface as a function of the flux linkage and rotor position $-i(\theta, \psi)$. As far as the current is estimated it is possible to evaluate the value of torque as a function of the phase current and position from $(4)-T(\theta, i)$. The torque function can be expressed from the energy (3) or coenergy (4) but as the original flux linkage surface is derived as a function of phase current, then it is easier to calculate the coenergy integral than to integrate the energy by flux.

The same calculations should be done for all three phases of the motor and the resulting torque is the sum of the torques produced by each phase of the motor:

$$
T=T_{A}+T_{B}+T_{C}
$$

Finally, the mechanical equations should be solved to evaluate the motor speed and position:

$$
\left.\begin{array}{l}
\Delta \omega=\frac{1}{J}\left(T-T_{\text {load }}\right) T_{P W M} \\
\Delta \theta=p_{p} \omega T_{P W M}
\end{array}\right\}
$$

where $J$ is the moment of inertia.

\section{E. Magnetisation Curves Processing}

To prepare the model for a real-time calculation the magnetisation curves should be processed in order to adapt data for fast $i(\theta, \psi)$ and $T(\theta, i)$ evaluation.

The original curves from Fig. 3 are usually represented by an array of $N$ points with specified values $i[n]$ and $\psi[n]$ where $n=1 \ldots N$. The data between the points are derived using cubic interpolation and an array of 100 points with fixed steps is obtained. A fixed step look-up table is used to speed up the calculation. A big number of points allows fast linear interpolation without decreasing the accuracy. The same procedure is done with the interpolation of intermediate curves by (1) for the whole revolution. The result of the interpolation is shown in Fig. 4.

The obtained surface is used to make an evaluation of the phase current and torque functions. Initially, the torque function is calculated. The torque produced can be evaluated by (4) for each of 100 points of current and angle using the following equation:

$$
\begin{aligned}
& T\left[N_{i}, N_{\theta}\right]= \\
& =p_{p} \frac{50}{2 \pi} \frac{I_{\max }}{100}\left(\sum_{n_{i}=1}^{N_{i}} \psi\left[n_{i}, n_{\theta}+1\right]-\psi\left[n_{i}, n_{\theta}-1\right]\right)
\end{aligned}
$$

This equation uses the difference between the numeric integrals of flux linkages by current for one curve in the control clockwise direction and another in the counter clockwise direction. The second coefficient $50 / 2 \pi$ is the angle difference between the curves and $I_{\max } / 100$ is the integration step size. After evaluating this look-up table the initialisation algorithm converts $\psi(\theta, i)$ surface into $i(\theta, \psi)$ look-up table by simple mathematical transformation.

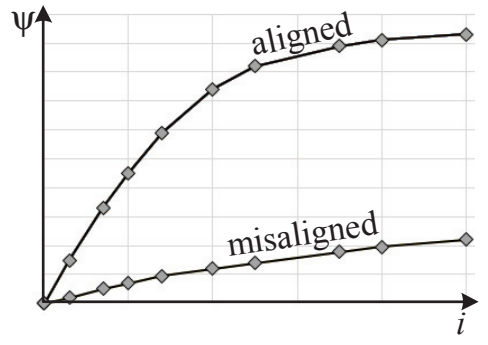

Fig. 3. Magnetisation curves for aligned and misaligned positions.

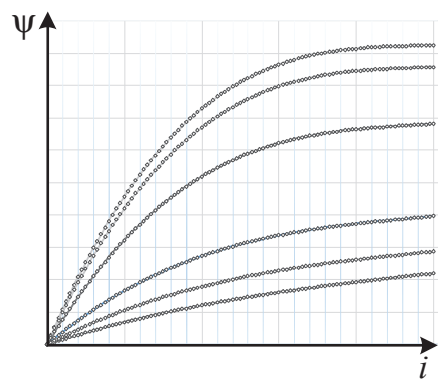

Fig. 4. Interpolation result for magnetisation curves (not all data are shown). 


\section{EXPERIMENTAL RESULTS}

The proposed model was implemented in $\mathrm{C}$ language. Its single step calculation lasts for less than $3000 \mathrm{CPU}$ cycles of approximately 15,000 cycles available at $10 \mathrm{kHz}$ of PWM. This allows PWM frequency to increase up to $40 \mathrm{kHz}$ if necessary. The code for examination of the model is shown in Fig. 5.

The results of the operation are shown in Fig. 6. It can be seen that phase $\mathrm{A}$ is switched on, when the winding inductance is minimum and approximately constant. Thus, the current rises with a constant slope. When the current reaches the reference value, the hysteresis current controller starts switching the phase off and on to stabilise the current. This lasts until the rotor angular position reaches the off point. The phase A goes to the off state, while phase B switches on. The current in phase A starts to reduce, but this time the teeth of the stator and rotor are aligned, thus the transient differs from the one during the switch on. At the beginning of the transient the motor is saturated and the current falls with high derivative. When its value passes the saturation knee, the motor goes out of the saturation and the phase inductance increases. The current slope decreases more smoothly when compared with the plot from Fig. $1 \mathrm{~b}$.

The switched reluctance motor can be operated in sensorless control system. An example of selfsensing control strategy is presented in Fig. 7. During off state the stator winding is put into operation for a single PWM cycle. The response in the phase current indicates instant phase inductance. This information can be used for position feedback.

\section{CONCLUSIONS}

The SRM drive model based on a look-up table has been suggested in this paper in order to be used in student coursework on electric drive control subjects. Due to the implementation of the look-up table for the magnetisation curve representation, the model accuracy and behaviour have been significantly increased in comparison with the linearised solution. The look-up table approach provides a fast calculation and low computation load because of the allocation of all of the complicated calculations at the initialisation stage. The model itself uses no more than 3000 CPU cycles. Thus, it is possible to debug control strategy in real-time at up to $40 \mathrm{kHz}$ PWM frequency. Operation of the model was verified using microcontrollers TMS320F2812 and TMS320F28335 from Texas Instruments and a Russian microcontroller K1921BK01T manufactured by NIIET (city of Voronezh).

This model can be used both for training of students and for the prototyping of control strategies in R\&D companies.

\section{REFERENCES}

[1] J. E. Mills, and D. F. Treagust, "Engineering education - Is problem based or project based learning the answer?" Australian Journal of Engineering Education, vol. 3, no. 2, pp. 2-16, 2003.

[2] B.K. Jesiek, and L.H. Jamieson, "The expansive (dis)integration of electrical engineering education," IEEE Access, vol. 5, pp. 4561-4573, March 2017.

[3] A. Anuchin, D. Savkin, Y. Khanova, and D. Grishchuk, "Real-time model for motor control coursework," in Proc. IEEE 5th Int. Conf. on Power Engineering, Energy and Electrical Drives POWERENG, 11-13 May 2015, Riga, pp. 427-430.
[4] R. Krishnan, Switched Reluctance Motor Drives: Modeling, Simulation, Analysis, Design, and Applications, Boca Raton, FL: CRC Press, 2001.

[5] P. Pillay, Y. Liu, W. Cai, and T. Sebastian, "Multiphase operation of switched reluctance motor drives," in Proc. IEEE 37th IAS Annual Meeting, 5-9 Oct. 1997, New Orleans, LA, USA, vol. 1, pp. 310-317.

[6] A. Anuchin, D. Grishchuk, A. Zharkov, Y. Prudnikova, and L. Gosteva, "Real-time model of switched reluctance drive for educational purposes," in Proc. 57th Int. Sci. Conf. on Power and Electrical Engineering of Riga Technical University RTUCON, 13-14 Oct. 2016, Riga, pp. 1-5.

[7] A. Fitzgerald, C. Kingsley, and S. Umans, Electric Machinery, 7th edn, New York: McGraw-Hill Education, 2014.

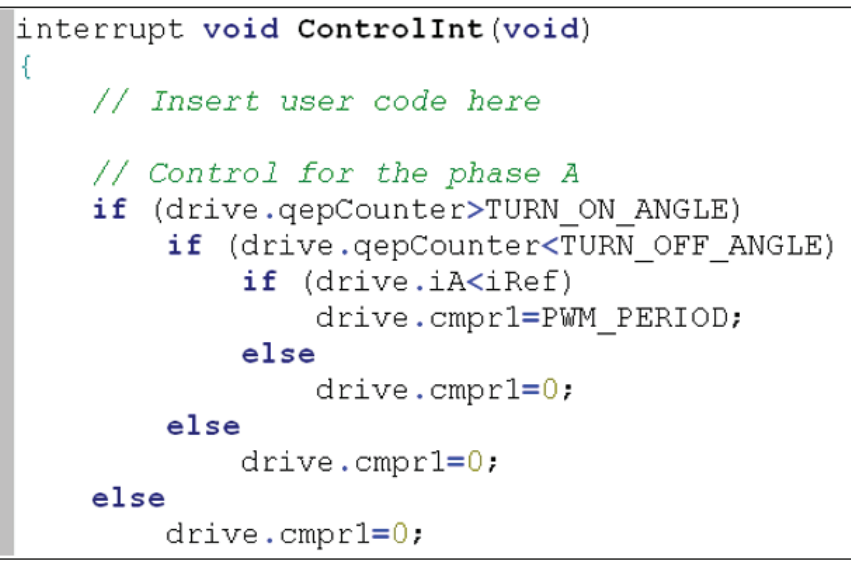

Fig. 5. Fragment of the code with the hysteresis current controller for the phase A (drive.qepCounter is the position from the virtual incremental encoder; TURN_ON_ANGLE is the constant for the turn-on angle; TURN_OFF_ANGLE is the constant for the turn-off angle; drive.iA is the code from the virtual ADC connected to the current sensor of the phase A; iRef is the variable for the current reference; drive.cmprl is the duty cycle for the phase A of the inverter; PWM_PERIOD is the maximum value of the PWM timer counter).

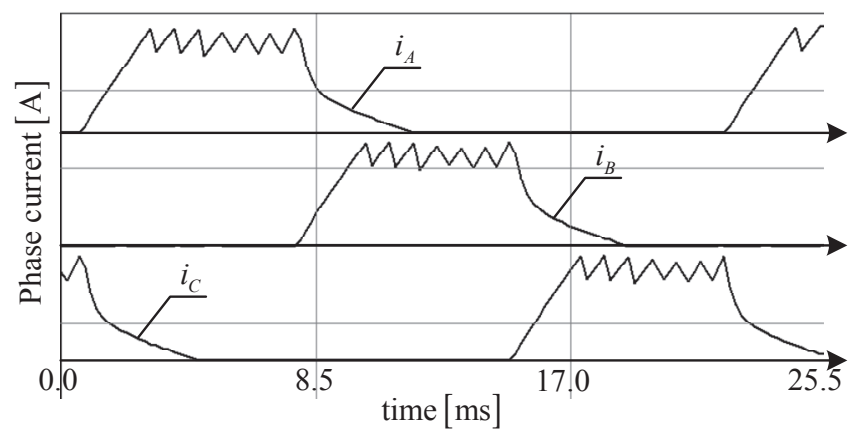

Fig. 6. Motor operation with the constant current reference $10 \mathrm{~A}$ at $680 \mathrm{rpm}$.

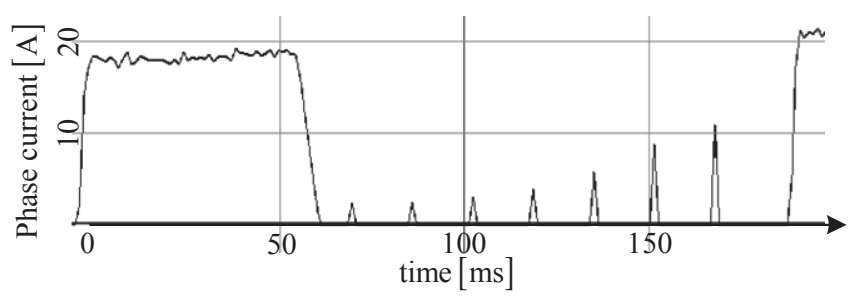

Fig. 7. Selfsensing operation of the SRD. 